Noname manuscript No.

(will be inserted by the editor)

\title{
Optimal Control of Impulsive Switched Systems with Minimum Subsystem Durations
}

\author{
Eunice Blanchard · Ryan Loxton · Volker Rehbock
}

Received: date / Accepted: date

\begin{abstract}
This paper presents a new computational approach for solving optimal control problems governed by impulsive switched systems. Such systems consist of multiple subsystems operating in succession, with possible instantaneous state jumps occurring when the system switches from one subsystem to another. The control variables are the subsystem durations and a set of system parameters influencing the state jumps. In contrast with most other papers on the control of impulsive switched systems, we do not require every potential subsystem to be active during the time horizon (it may be optimal to delete certain subsystems, especially when the optimal number of switches is unknown). However, any active subsystem must be active for a minimum non-negligible duration of time. This restriction leads to a disjoint feasible region for the subsystem durations. The problem of choosing the subsystem durations and the system parameters to minimize a given cost function is a non-standard optimal control problem that cannot be solved using conventional techniques. By combining a time-scaling transformation and an exact penalty method, we develop a computational algorithm for solving this problem. We then demonstrate the effectiveness of this algorithm by considering a numerical example on the optimization of shrimp harvesting operations.
\end{abstract}

Keywords Optimal Control · Impulsive System • Switched System • Time-Scaling Transformation • Exact Penalty Function

\section{Introduction}

An impulsive switched system is a dynamic system whose state and state dynamics undergo instantaneous changes at certain times in the time horizon. These times, called jump times or switching times, are usually decision variables to be chosen optimally by the system operator. In practical applications, the goal is to optimize the impulsive switched system by manipulating the jump times, as well as other control parameters influencing the state dynamics and/or state jumps, to achieve optimal system performance. Impulsive switched systems arise in many practical applications including switching DC-DC power converters [1,12], shrimp harvesting [20] and communication security [5].

R. Loxton is supported by an International Young Scientists Research Fellowship from the National Natural Science Foundation of China (grant no. 11350110208), and an International Collaboration Grant from the State Key Laboratory of Industrial Control Technology at Zhejiang University, China (grant no. ICT1301).

E. Blanchard

Department of Mathematics and Statistics, Curtin University, Australia

E-mail: anitmani@gmail.com

R. Loxton

Department of Mathematics and Statistics, Curtin University, Australia

Institute of Cyber-Systems and Control, Zhejiang University, China

E-mail: r.loxton@curtin.edu.au

V. Rehbock

Department of Mathematics and Statistics, Curtin University, Australia

E-mail: v.rehbock@curtin.edu.au 
Consider an impulsive switched system consisting of $N$ subsystems operating in succession. Let $\tau_{i}$ denote the $i^{t h}$ switching time. Then $\tau_{i}, i=1, \ldots, N$, satisfy the following ordering constraints:

$$
0=\tau_{0} \leq \tau_{1} \leq \tau_{2} \leq \cdots \leq \tau_{N}
$$

In the literature on the optimal control of impulsive switched systems, the inequalities in (1) are usually the only constraints imposed on the switching times (see, for example, references $[4,5,10$, 15, 17-19]). However, the constraints in (1) do not preclude very short operating durations for some subsystems. In reality, it is physically impossible to switch too frequently, and hence there is often a minimum duration for which a subsystem must be active. A first attempt at addressing this issue was made in [16], where a penalty on short subsystem durations was added to the objective function. However, this is only a heuristic approach, and there is no guarantee that it yields optimal (or even feasible) results for general impulsive switched systems.

Reference [10] considers an optimal control problem governed by an impulsive system in which the state variables (but not the state dynamics) experience abrupt jumps at a finite number of jump times. The magnitudes of the state jumps are controlled through a set of system parameters. To solve the problem in [10], the authors developed a transformation procedure that involves introducing a new time variable and mapping the jump times (which are decision variables to be chosen optimally) into fixed points in a new time horizon. The resulting problem can then be solved using gradientbased optimal control software such as MISER 3.3 [2]. The technique designed in [10] usually performs reasonably well in practice, although it is only capable of finding locally optimal solutions. In [18], this limitation was overcome by combining the gradient-based optimization technique in [10] with a filled function method for finding globally optimal solutions.

Although the methods in $[10,18]$ usually work well, they do not have the capability to preclude extremely short operating durations. In fact, the numerical example in [10] has solutions in which some of the subsystem durations become very short and disappear altogether when the number of allowed state jumps is increased. Due to the formulation of the problem class in $[10,18]$, when a subsystem duration becomes zero during the optimization process, the two corresponding state jumps still occur. But it would be more appropriate in this situation if one of these state jumps is deleted, so that only one jump occurs at the common jump time. The methods in $[10,18]$ do not have this capability: they instead impose "multi-jumps" when two or more switching times coincide. This challenge was overcome in [11] by combining the time-scaling transformation from $[10,18]$ with a penalty technique. The numerical results in [11] demonstrate that subsystems can be deleted in the optimal solution without the occurrence of multiple state jumps.

More recently in [8], a new algorithm for solving optimal control problems governed by impulsive switched systems was developed. This algorithm is based on a new approach to applying the timescaling transformation, as well as a new method for computing the cost function's gradient via the solution of an auxiliary dynamic system. The disadvantage with the algorithm in [8] is that every potential subsystem is assumed to operate for a positive duration. Hence, unlike in [11], there is no scope to remove non-optimal subsystems.

In this paper, we assume that each subsystem either does not operate (duration of zero) or operates for a minimum non-negligible amount of time (duration is no less than a specified minimum positive number). This requires that adjacent switching times satisfy the constraint $\tau_{i}-\tau_{i-1} \in\{0\} \cup\left[\epsilon_{i}, \infty\right)$, where $\tau_{i}$ is as defined in (1) and $\epsilon_{i}>0$ is the minimum duration of the $i^{\text {th }}$ subsystem. Unlike (1), this constraint defines a disjoint region for the subsystem durations, thus causing problems for standard optimization algorithms such as sequential quadratic programming $[13,14]$. In this paper, we introduce a novel exact penalty function to overcome this challenge.

The class of problems considered in this paper is more general than the class of problems considered in [11]. In particular, we allow canonical state constraints, whereas no state constraints are considered in [11]. Furthermore, we develop a transformation procedure that transforms the problem with disjoint feasible region into an equivalent standard dynamic optimization problem. In contrast, the approach in [11] is based on an approximation scheme and does not maintain equivalence.

This paper is organized as follows. In Section 2, we define the optimal control problem under consideration. Then, in Section 3, we use the time-scaling technique [8] to map the variable jump times to fixed integers in a new time horizon. After this, we implement another transformation by introducing new binary variables. However, the constraints on the binary variables define a disjoint feasible region, which poses a challenge for standard optimal control software such as MISER 3.3. Therefore, we adopt an exact penalty approach in Section 4 to transform the problem into a sequence of unconstrained problems with fixed jump times. Each of these unconstrained problems is a smooth 
impulsive optimal control problem that can be solved effectively by MISER 3.3. Our numerical example in Section 5 provides evidence that the proposed method is effective and efficient.

\section{Problem Formulation}

Consider the following impulsive switched system:

$$
\dot{\boldsymbol{x}}(t)=\boldsymbol{f}^{i}(\boldsymbol{x}(t), \boldsymbol{\zeta}), \quad t \in\left(\tau_{i-1}, \tau_{i}\right), \quad i=1, \ldots, N,
$$

with jump conditions

$$
\boldsymbol{x}\left(\tau_{i}^{+}\right)= \begin{cases}\boldsymbol{x}^{0}, & \text { if } i=0, \\ \boldsymbol{x}\left(\tau_{i}^{-}\right)+\phi^{i}\left(\boldsymbol{x}\left(\tau_{i}^{-}\right), \boldsymbol{\zeta}\right), & \text { if } i \in\{1, \ldots, N\} \text { and } \tau_{i}-\tau_{i-1} \geq \epsilon_{i},\end{cases}
$$

where

- $\boldsymbol{x}(t) \in \mathbb{R}^{n}$ is the state at time $t$

- $\zeta \in \mathbb{R}^{r}$ is a vector of control parameters;

- $\boldsymbol{x}^{0} \in \mathbb{R}^{n}$ is a given initial state;

- $\tau_{i}, i=1, \ldots, N-1$, are jump or switching times, with $\tau_{0}=0$ and $\tau_{N}=T$;

- $T>0$ is a given terminal time;

- $\epsilon_{i}>0$ is the given minimum duration of the $i^{\text {th }}$ subsystem;

- $\boldsymbol{f}^{i}: \mathbb{R}^{n} \times \mathbb{R}^{r} \rightarrow \mathbb{R}^{n}$ and $\phi^{i}: \mathbb{R}^{n} \times \mathbb{R}^{r} \rightarrow \mathbb{R}^{n}, i=1, \ldots, N$, are given functions assumed to be continuously differentiable.

Note that the positive and negative superscripts in $(3 \mathrm{~b})$ mean $\boldsymbol{x}\left(\tau^{ \pm}\right)=\lim _{t \rightarrow \tau^{ \pm}} \boldsymbol{x}(t)$. Note also that if $\tau_{i}=T$ in (3b), then $\tau_{i}^{+}=T$ by convention.

System (2)-(3) consists of $N$ potential subsystems operating in succession. The dynamic behaviour of the $i^{t h}$ subsystem is governed by the function $f^{i}$. At the switching times $\tau_{i}, i=1, \ldots, N-1$, the system changes from one subsystem to another, and this causes an instantaneous jump in the system state according to $(3 \mathrm{~b})$.

Note that if $\tau_{i-1}$ and $\tau_{i}$ coincide (i.e. $\tau_{i-1}=\tau_{i}$ ), then the $i^{\text {th }}$ subsystem does not operate. This is allowed, as running every potential subsystem may not be optimal. The condition $\tau_{i}-\tau_{i-1} \geq \epsilon_{i}$ in (3b) ensures that the state jump occurring at the end of a certain subsystem is only imposed if that particular subsystem runs for a non-negligible amount of time. This is different to the conventional impulsive switched systems considered in the literature (see, for example, $[10,17,18]$ ), which impose state jumps even for subsystems that do not operate. For example, if $\tau_{1}=\tau_{2}$ in a conventional impulsive switched system, then a "double jump" - one for subsystem 1 and another for subsystem 2will be imposed at $t=\tau_{1}=\tau_{2}$, even though subsystem 2 does not actually operate. This is usually not an accurate reflection of the real system under consideration.

The control parameter vector $\zeta=\left[\zeta_{1}, \ldots, \zeta_{r}\right]^{\top} \in \mathbb{R}^{r}$ is subject to the following bound constraints:

$$
a_{j} \leq \zeta_{j} \leq b_{j}, \quad j=1, \ldots, r,
$$

where $a_{j}$ and $b_{j}$ are given constants such that $a_{j}<b_{j}$. Let $\mathcal{Z}$ denote the set of all $\zeta \in \mathbb{R}^{r}$ satisfying (4).

We also have the following constraints on the switching times:

$$
\tau_{i}-\tau_{i-1} \in\{0\} \cup\left[\epsilon_{i}, T\right], \quad i=1, \ldots, N .
$$

Thus, either subsystem $i$ runs for a duration of at least $\epsilon_{i}$ time units, or it does not run at all. This means that it is possible to "delete" certain subsystems if it is optimal to do so, which may be necessary when the optimal number of switches is unknown. Clearly, constraint (5) is more complex than constraint (1) given earlier, which is a simple ordering constraint on the switching times. In particular, (1) is convex, but (5) is non-convex. In fact, imposing (5) leads to a disjoint feasible region for the subsystem durations.

Let $\Gamma$ denote the set of all $\boldsymbol{\tau}=\left[\tau_{1}, \ldots, \tau_{N-1}\right]^{\top} \in \mathbb{R}^{N-1}$ satisfying constraint (5). Furthermore, let $\boldsymbol{x}(\cdot \mid \boldsymbol{\tau}, \boldsymbol{\zeta})$ denote the right-continuous solution of (2)-(3) corresponding to the given pair $(\boldsymbol{\tau}, \boldsymbol{\zeta}) \in \Gamma \times \mathcal{Z}$. 
We suppose that the system is subject to the following canonical constraints:

$$
G_{j}(\boldsymbol{\tau}, \boldsymbol{\zeta})=\sum_{i=1}^{N} \widehat{\Phi}_{j, i}\left(\tau_{i}-\tau_{i-1}, \boldsymbol{x}\left(\tau_{i} \mid \boldsymbol{\tau}, \boldsymbol{\zeta}\right), \boldsymbol{\zeta}\right) \begin{cases}=0, & j=1, \ldots, q_{e} \\ \geq 0, & j=q_{e}+1, \ldots, q\end{cases}
$$

where

$$
\widehat{\Phi}_{j, i}\left(\tau_{i}-\tau_{i-1}, \boldsymbol{x}\left(\tau_{i} \mid \boldsymbol{\tau}, \boldsymbol{\zeta}\right), \boldsymbol{\zeta}\right)= \begin{cases}\Phi_{j, i}\left(\boldsymbol{x}\left(\tau_{i} \mid \boldsymbol{\tau}, \boldsymbol{\zeta}\right), \boldsymbol{\zeta}\right), & \text { if } \tau_{i}-\tau_{i-1} \geq \epsilon_{i} \\ 0, & \text { if } \tau_{i}-\tau_{i-1}=0\end{cases}
$$

and $\Phi_{j, i}: \mathbb{R}^{n} \times \mathbb{R}^{r} \rightarrow \mathbb{R}$ are given functions assumed to be continuously differentiable with respect to each of their arguments. Here, the definition of $\widehat{\Phi}_{j, i}$ ensures that each distinct switch contributes exactly once to the $j^{\text {th }}$ constraint. More specifically, if $\tau_{i}=\tau_{i-1}$, then the $i^{\text {th }}$ term in the summation on the right-hand side of (6) is set equal to zero to avoid "doubling up" when multiple switching times coincide. For example, if $\tau_{1}=\tau_{2}>0$, then $\widehat{\Phi}_{j, 1}=\Phi_{j, 1}$, but $\widehat{\Phi}_{j, 2}=0$; that is, there is no contribution from the second subsystem to the $j^{\text {th }}$ constraint because the second subsystem does not actually operate.

The system cost function defined below is assumed to take the same canonical form as the constraints in (6):

$$
G_{0}(\boldsymbol{\tau}, \boldsymbol{\zeta})=\sum_{i=1}^{N} \widehat{\Phi}_{0, i}\left(\tau_{i}-\tau_{i-1}, \boldsymbol{x}\left(\tau_{i} \mid \boldsymbol{\tau}, \boldsymbol{\zeta}\right), \boldsymbol{\zeta}\right)
$$

where $\widehat{\Phi}_{0, i}$ is defined in a similar way to $\widehat{\Phi}_{j, i}$ in $(7)$.

Note that the canonical functions in (7) and (8) are discontinuous functions of the subsystem durations. This formulation is in contrast to the usual definition of canonical functions in optimal control $[2,6]$, and has, to the best of our knowledge, not previously been considered in the literature.

Our problem is to choose $(\boldsymbol{\tau}, \boldsymbol{\zeta}) \in \Gamma \times \mathcal{Z}$ to minimize the cost function given by (8) subject to the governing impulsive switched system given by (2) and (3), the bounds (4), the non-convex constraints (5) and the canonical constraints (6). We refer to this problem as Problem A.

\section{Problem Transformation}

\subsection{Time-Scaling Transformation}

It is well known in computational optimal control that standard numerical optimization algorithms are not effective at optimizing variable switching times $[3,7]$. Thus, in this section, we will apply the time-scaling transformation described in $[6,8,10]$ to map the variable switching times to fixed times in a new time horizon. This yields an equivalent problem in which the variable switching times are replaced by conventional decision parameters.

We first introduce a new time variable $s \in[0, N]$ and relate $s$ to $t$ through the following differential equation:

$$
\dot{t}(s)=\sum_{i=1}^{N} \theta_{i} \chi_{[i-1, i)}(s), \quad t(0)=0,
$$

and

$$
t(N)=T,
$$

where $\theta_{i}=\tau_{i}-\tau_{i-1}$ is the duration of the $i^{t h}$ subsystem and, for a given interval $I, \chi_{I}(s)$ is the corresponding indicator function defined by

$$
\chi_{I}(s)= \begin{cases}1, & \text { if } s \in I \\ 0, & \text { otherwise }\end{cases}
$$

Constraints (5) can be equivalently expressed in terms of the following inequalities:

$$
\left(\tau_{i}-\tau_{i-1}\right)\left(\epsilon_{i}-\tau_{i}+\tau_{i-1}\right) \leq 0, \quad i=1, \ldots, N
$$

and

$$
\tau_{i} \geq \tau_{i-1}, \quad i=1, \ldots, N .
$$


Based on the definition of $\theta_{i}$, constraints (10) and (11) become:

$$
\theta_{i}\left(\epsilon_{i}-\theta_{i}\right) \leq 0, \quad i=1, \ldots, N
$$

and

$$
\theta_{i} \geq 0, \quad i=1, \ldots, N .
$$

For $s \in[i-1, i]$, it follows from (9) that

$$
t(s)=\int_{0}^{s} \dot{t}(\eta) d \eta=\sum_{k=1}^{i-1} \theta_{k}+\theta_{i}(s-i+1) .
$$

Thus, for each $i=0, \ldots, N$,

$$
t(i)=\sum_{k=1}^{i} \theta_{k}=\sum_{k=1}^{i}\left(\tau_{k}-\tau_{k-1}\right)=\tau_{i}
$$

In particular, $t(N)=\tau_{N}=T$, as required by equation (9b).

Let $\widetilde{\boldsymbol{x}}(s)=\boldsymbol{x}(t(s))$, where $t(s)$ is the solution of the differential equation (9a). Since $\theta_{i} \geq 0$, it is clear that $t$ is a non-decreasing function of $s$. Thus, if $s \in(i-1, i)$, then

$$
t(s) \in[t(i-1), t(i)]=\left[\theta_{1}+\cdots+\theta_{i-1}, \theta_{1}+\cdots+\theta_{i}\right] .
$$

In fact, if $\theta_{i}>0$, then

$$
t(s) \in\left(\theta_{1}+\cdots+\theta_{i-1}, \theta_{1}+\cdots+\theta_{i}\right), \quad s \in(i-1, i),
$$

and thus

$$
\frac{d}{d s}\{\widetilde{\boldsymbol{x}}(s)\}=\frac{d}{d s}\{\boldsymbol{x}(t(s))\}=\dot{t}(s) \dot{\boldsymbol{x}}(t(s))=\theta_{i} \boldsymbol{f}^{i}(\widetilde{\boldsymbol{x}}(s), \boldsymbol{\zeta}), \quad s \in(i-1, i) .
$$

On the other hand, if $\theta_{i}=0$, then $t(s)=\theta_{1}+\cdots+\theta_{i-1}$ for all $s \in(i-1, i)$, and thus

$$
\frac{d}{d s}\{\widetilde{\boldsymbol{x}}(s)\}=\frac{d}{d s}\{\boldsymbol{x}(t(s))\}=\frac{d}{d s}\left\{\boldsymbol{x}\left(\theta_{1}+\cdots+\theta_{i-1}\right)\right\}=\mathbf{0}, \quad s \in(i-1, i) .
$$

Combining (16a) and (16b) shows that, under the time-scaling transformation, the system dynamics in Problem A become

$$
\dot{\widetilde{\boldsymbol{x}}}(s)=\theta_{i} \boldsymbol{f}^{i}(\widetilde{\boldsymbol{x}}(s), \boldsymbol{\zeta}), \quad s \in(i-1, i), \quad i=1, \ldots, N .
$$

Since the time-scaling transformation maps $s=i$ to $t=\tau_{i}$ (see (14)), the state jumps in the new time horizon occur at the fixed times $s=1, \ldots, N$. If $\theta_{i}>0$, then $\theta_{i} \geq \epsilon_{i}$, and thus $t(i)-t(i-1)=\theta_{i} \geq \epsilon_{i}$. It therefore follows from $(3 \mathrm{~b})$ that

$$
\widetilde{\boldsymbol{x}}\left(i^{+}\right)=\widetilde{\boldsymbol{x}}\left(i^{-}\right)+\phi^{i}\left(\widetilde{\boldsymbol{x}}\left(i^{-}\right), \boldsymbol{\zeta}\right) .
$$

If, on the other hand, $\theta_{i}=0$, then $t(i)-t(i-1)=\theta_{i}=0$. Hence,

$$
\widetilde{\boldsymbol{x}}\left(i^{+}\right)=\widetilde{\boldsymbol{x}}\left(i^{-}\right) \text {. }
$$

Consequently, the jump conditions (3) become

$$
\widetilde{\boldsymbol{x}}\left(i^{+}\right)= \begin{cases}\boldsymbol{x}^{0}, & \text { if } i=0, \\ \widetilde{\boldsymbol{x}}\left(i^{-}\right)+\boldsymbol{\phi}^{i}\left(\widetilde{\boldsymbol{x}}\left(i^{-}\right), \boldsymbol{\zeta}\right), & \text { if } i \in\{1, \ldots, N\} \text { and } \theta_{i} \geq \epsilon_{i}, \\ \widetilde{\boldsymbol{x}}\left(i^{-}\right), & \text {if } i \in\{1, \ldots, N\} \text { and } \theta_{i}=0 .\end{cases}
$$

Note that by convention, $i^{+}=N$ when $i=N$ in (18). Let $\widetilde{\boldsymbol{x}}(\cdot \mid \boldsymbol{\theta}, \boldsymbol{\zeta})$ denote the unique right-continuous solution of (17) and (18) corresponding to $\boldsymbol{\theta}=\left[\theta_{1}, \ldots, \theta_{N}\right]^{\top} \in \mathbb{R}^{N}$ and $\boldsymbol{\zeta}=\left[\zeta_{1}, \ldots, \zeta_{r}\right]^{\top} \in \mathbb{R}^{r}$. Under the time-scaling transformation, the canonical constraints given by (6) become

$$
\widetilde{G}_{j}(\boldsymbol{\theta}, \boldsymbol{\zeta})=\sum_{i=1}^{N} \widehat{\Phi}_{j, i}\left(\theta_{i}, \widetilde{\boldsymbol{x}}(i \mid \boldsymbol{\theta}, \boldsymbol{\zeta}), \boldsymbol{\zeta}\right) \begin{cases}=0, & j=1, \ldots, q_{e}, \\ \geq 0, & j=q_{e}+1, \ldots, q,\end{cases}
$$


where $\widehat{\Phi}_{j, i}$ is defined by (7). Problem A is thus transformed into the following problem: Choose $(\boldsymbol{\theta}, \boldsymbol{\zeta}) \in \mathbb{R}^{N} \times \mathbb{R}^{r}$ to minimize the transformed cost function

$$
\widetilde{G}_{0}(\boldsymbol{\theta}, \boldsymbol{\zeta})=\sum_{i=1}^{N} \widehat{\Phi}_{0, i}\left(\theta_{i}, \widetilde{\boldsymbol{x}}(i \mid \boldsymbol{\theta}, \boldsymbol{\zeta}), \boldsymbol{\zeta}\right)
$$

subject to the dynamics (9) and (17), the constraints (4), (12) and (19) and the jump conditions (18). We refer to this problem as Problem B.

Problems A and B are mathematically equivalent. Thus, a solution of Problem A can be used to generate a solution of Problem B, and vice versa. Note that the variable jump times in Problem A have been replaced by fixed jump times in Problem B. This makes Problem B much easier to deal with from a computational point of view, as it is well known that variable jump times cause numerical difficulties $[6,8,10]$. However, Problem B still cannot be solved using conventional impulsive control techniques. This is because the jump conditions (18) and the canonical functions in (19) and (20) are expressed as discontinuous piecewise functions of $\theta_{i}$. In fact, (18) can be written as follows:

$$
\widetilde{\boldsymbol{x}}\left(i^{+}\right)= \begin{cases}\boldsymbol{x}^{0}, & \text { if } i=0, \\ \widetilde{\boldsymbol{x}}\left(i^{-}\right)+\chi_{\left[\epsilon_{i}, \infty\right)}\left(\theta_{i}\right) \phi^{i}\left(\widetilde{\boldsymbol{x}}\left(i^{-}\right), \boldsymbol{\zeta}\right), & \text { if } i \in\{1, \ldots, N\},\end{cases}
$$

where

$$
\chi_{\left[\epsilon_{i}, \infty\right)}\left(\theta_{i}\right)= \begin{cases}1, & \text { if } \theta_{i} \geq \epsilon_{i} \\ 0, & \text { otherwise. }\end{cases}
$$

Clearly, the presence of the indicator function $\chi_{\left[\epsilon_{i}, \infty\right)}$ causes the jump conditions (21) to be discontinuous. In [11], this difficulty was tackled by approximating the indicator function by a continuously differentiable function that depends on a smoothing parameter. However, to achieve sufficient accuracy, the value of the smoothing parameter must be reduced to the point where it leads to numerical difficulties.

In the next section, we propose a new approach that involves introducing binary decision variables to transform the jump conditions and the canonical functions into smooth forms. The advantage of this new approach is that it yields an equivalent problem, not just an approximation.

\subsection{Transforming the Jump Conditions and Canonical Functions}

Let $v_{i}, i=1, \ldots, N$, be new binary decision variables defined as follows:

$$
v_{i}= \begin{cases}1, & \text { if } \theta_{i} \geq \epsilon_{i} \\ 0, & \text { if } \theta_{i}=0\end{cases}
$$

Using these binary variables, the jump conditions in (18) can be written in a more compact form as

$$
\widetilde{\boldsymbol{x}}\left(i^{+}\right)= \begin{cases}\boldsymbol{x}^{0}, & \text { if } i=0, \\ \widetilde{\boldsymbol{x}}\left(i^{-}\right)+v_{i} \phi^{i}\left(\widetilde{\boldsymbol{x}}\left(i^{-}\right), \boldsymbol{\zeta}\right), & \text { if } i \in\{1, \ldots, N\} .\end{cases}
$$

As in (18), we use the convention $N^{+}=N$ here.

Let $\boldsymbol{v}=\left[v_{1}, v_{2}, \ldots, v_{N}\right]^{\top}$. Furthermore, let $\widetilde{\boldsymbol{x}}(\cdot \mid \boldsymbol{v}, \boldsymbol{\theta}, \boldsymbol{\zeta})$ denote the right-continuous solution of (17) and (23) corresponding to $(\boldsymbol{v}, \boldsymbol{\theta}, \boldsymbol{\zeta})$. Then the canonical constraints in (19) can be written as

$$
\widetilde{G}_{j}(\boldsymbol{v}, \boldsymbol{\theta}, \boldsymbol{\zeta})=\sum_{i=1}^{N} v_{i} \Phi_{j, i}(\widetilde{\boldsymbol{x}}(i \mid \boldsymbol{v}, \boldsymbol{\theta}, \boldsymbol{\zeta}), \boldsymbol{\zeta}) \begin{cases}=0, & j=1, \ldots, q_{e}, \\ \geq 0, & j=q_{e}+1, \ldots, q .\end{cases}
$$

Standard optimization algorithms such as interior-point methods and sequential quadratic programming (see $[13,14]$ ) cannot handle binary variables in the form of $(22)$. Therefore, to proceed, we drop the binary requirements and consider each $v_{i}$ as a continuous optimization variable subject to the following constraints:

$$
0 \leq v_{i} \leq 1, \quad i=1, \ldots, N
$$

and

$$
g_{i}\left(v_{i}\right)=v_{i}\left(1-v_{i}\right) \leq 0, \quad i=1, \ldots, N
$$


It is clear that (25) and (26) imply $v_{i} \in\{0,1\}$. However, we also need to ensure that $v_{i}$ is consistent with the definition given in (22). Thus, we impose the following additional constraints:

$$
h_{i}\left(v_{i}, \theta_{i}\right)=\left(\epsilon_{i}-\theta_{i}\right) v_{i} \leq 0, \quad i=1, \ldots, N
$$

and

$$
H_{i}\left(v_{i}, \theta_{i}\right)=\theta_{i}\left(1-v_{i}\right) \leq 0, \quad i=1, \ldots, N .
$$

We now prove that definition (22) is equivalent to (25)-(28).

Theorem 1 Suppose that $v_{i}, i=1, \ldots, N$, satisfy constraints (25) and (26). Then for each integer $i \in\{1, \ldots, N\}$, (22) holds if only if (27) and (28) hold.

Proof First, suppose that (22) is satisfied. Then $v_{i}=0$ implies $\theta_{i}=0$ and $h_{i}\left(v_{i}, \theta_{i}\right)=0$. Furthermore, $\theta_{i}=0$ implies $H_{i}\left(v_{i}, \theta_{i}\right)=0$.

Similarly, $v_{i}=1$ implies $\theta_{i} \geq \epsilon_{i}, h_{i}\left(v_{i}, \theta_{i}\right)=\epsilon_{i}-\theta_{i} \leq 0$ and $H_{i}\left(v_{i}, \theta_{i}\right)=0$. Therefore, $(27)$ and (28) are satisfied.

Conversely, suppose inequalities (27) and (28) are satisfied. By (25) and (26), $v_{i} \in\{0,1\}$. If $v_{i}=0$, then by (28),

$$
v_{i}=0 \quad \Longrightarrow \quad \theta_{i}=\theta_{i}\left(1-v_{i}\right) \leq 0 .
$$

Since $\theta_{i}$, the duration of the $i^{\text {th }}$ subinterval, is non-negative, (29) implies that $\theta_{i}$ must be equal to zero. Thus, if $v_{i}=0$, then $\theta_{i}=0$ as required by $(22 \mathrm{~b})$.

On the other hand, if $v_{i}=1$, then by (27),

$$
v_{i}=1 \quad \Longrightarrow \quad \epsilon_{i}-\theta_{i}=\left(\epsilon_{i}-\theta_{i}\right) v_{i} \leq 0 \quad \Longrightarrow \quad \theta_{i} \geq \epsilon_{i} .
$$

Hence, if $v_{i}=1$, then $\theta_{i} \geq \epsilon_{i}$ as required by (22a). This completes the proof.

Remark 1 Recall that constraints (12a) ensure that each active subsystem operates for at least the length of its minimum duration. We now show that, with the new constraints (27) and (28) in place, constraints (12a) actually become redundant. Suppose that (12b) and (25)-(28) are satisfied and $0<\theta_{i}<\epsilon_{i}$. If $v_{i}=0$, then (12b) and (28) imply $\theta_{i}=0$, which is a contradiction. If $v_{i}=1$, then (27) implies $\epsilon_{i}-\theta_{i} \leq 0$, which is also a contradiction. Thus, when (12b) and (25)-(28) hold, it is impossible for $\theta_{i}$ to lie in the open interval $\left(0, \epsilon_{i}\right)$. This implies that $(12 \mathrm{a})$ is redundant.

With this remark in mind, we now define a new problem as follows: Choose $(\boldsymbol{v}, \boldsymbol{\theta}, \boldsymbol{\zeta}) \in \mathbb{R}^{N} \times \mathbb{R}^{N} \times$ $\mathbb{R}^{r}$ to minimize the cost function

$$
\widetilde{G}_{0}(\boldsymbol{v}, \boldsymbol{\theta}, \boldsymbol{\zeta})=\sum_{i=1}^{N} v_{i} \Phi_{0, i}(\widetilde{\boldsymbol{x}}(i \mid \boldsymbol{v}, \boldsymbol{\theta}, \boldsymbol{\zeta}), \boldsymbol{\zeta})
$$

subject to the dynamics given by (9) and (17), the jump conditions given by (23), the constraints given by (12b) and (25)-(28), the canonical constraints given by (24) and the bounds given by (4). We refer to this problem as Problem C.

By transforming Problem B into Problem C, we obtain smooth jump conditions and smooth canonical functions. The remaining difficulty is that, as the constraints (25) and (26) define a disjoint feasible region for $v_{i}, i=1, \ldots, N$, standard numerical optimization algorithms will struggle to find an optimal solution. In the next section, we introduce a penalty method to overcome this difficulty.

\section{An Exact Penalty Method}

The exact penalty approach involves forming a new objective function by adding terms based on the constraints to the objective. With this approach, Problem $\mathrm{C}$, a constrained optimization problem, is transformed into an approximate unconstrained problem that can be readily solved using the optimal control software MISER 3.3. MISER 3.3 automatically calculates the gradient of the objective function using a numerical procedure that involves integrating a costate system backwards in time. For more details, see $[6,10]$. 
The constraint violation is defined by:

$$
\begin{array}{r}
\Delta(\boldsymbol{v}, \boldsymbol{\theta}, \boldsymbol{\zeta})=\sum_{j=1}^{q_{e}}\left[\widetilde{G}_{j}(\boldsymbol{v}, \boldsymbol{\theta}, \boldsymbol{\zeta})\right]^{2}+\sum_{j=q_{e}+1}^{q}\left[\min \left\{0, \widetilde{G}_{j}(\boldsymbol{v}, \boldsymbol{\theta}, \boldsymbol{\zeta})\right\}\right]^{2} \\
+\sum_{i=1}^{N}\left[\max \left\{0, g_{i}\left(v_{i}\right)\right\}\right]^{2}+\sum_{i=1}^{N}\left[\max \left\{0, h_{i}\left(v_{i}, \theta_{i}\right)\right\}\right]^{2} \\
+\sum_{i=1}^{N}\left[\max \left\{0, H_{i}\left(v_{i}, \theta_{i}\right)\right\}\right]^{2}+[t(N)-T]^{2} .
\end{array}
$$

Note that $\Delta(\boldsymbol{v}, \boldsymbol{\theta}, \boldsymbol{\zeta})=0$ if and only if constraints (9b), (24) and (26)-(28) are satisfied.

Using the strategy introduced in $[3,9,21,22]$, an exact penalty function $\widehat{J}_{\sigma}(\boldsymbol{v}, \boldsymbol{\theta}, \boldsymbol{\zeta}, \lambda)$ is defined as follows:

$$
\widehat{J}_{\sigma}(\boldsymbol{v}, \boldsymbol{\theta}, \boldsymbol{\zeta}, \lambda)= \begin{cases}\widetilde{G}_{0}(\boldsymbol{v}, \boldsymbol{\theta}, \boldsymbol{\zeta}), & \text { if } \lambda=0 \text { and } \Delta(\boldsymbol{v}, \boldsymbol{\theta}, \boldsymbol{\zeta})=0 \\ \widetilde{G}_{0}(\boldsymbol{v}, \boldsymbol{\theta}, \boldsymbol{\zeta})+\lambda^{-\alpha} \Delta(\boldsymbol{v}, \boldsymbol{\theta}, \boldsymbol{\zeta})+\sigma \lambda^{\beta}, & \text { if } \lambda>0, \\ +\infty, & \text { otherwise, }\end{cases}
$$

where

- $\lambda$ is a new decision variable;

- $\sigma>0$ is the penalty parameter;

- $\alpha$ and $\beta$ are positive constants satisfying $1 \leq \beta \leq \alpha$.

The new decision variable $\lambda$ is subject to the following bounds:

$$
0 \leq \lambda \leq \tilde{\lambda}
$$

where $\tilde{\lambda}>0$ is a small positive number.

We now define the following unconstrained penalty problem: Choose $(\boldsymbol{v}, \boldsymbol{\theta}, \boldsymbol{\zeta}) \in \mathbb{R}^{N} \times \mathbb{R}^{N} \times \mathbb{R}^{r}$ and $\lambda \in \mathbb{R}$ to minimize $\widehat{J}_{\sigma}(\boldsymbol{v}, \boldsymbol{\theta}, \boldsymbol{\zeta}, \lambda)$ subject to the dynamics given by (9a) and (17), the jump conditions (23) and the bounds given by (4), (12b), (25) and (30). We refer to this problem as Problem D.

Note that when the penalty parameter $\sigma$ is large, the third term $\sigma \lambda^{\beta}$ in $\widehat{J}_{\sigma}$ forces $\lambda$ to be small, thus causing the second term $\lambda^{-\alpha} \Delta(\boldsymbol{v}, \boldsymbol{\theta}, \boldsymbol{\zeta})$ to severely penalize any constraint violations. When the penalty parameter $\sigma$ is sufficiently large, any solution of the penalty problem (i.e. Problem D) is also an optimal solution of Problem C [9,21-23].

Problem D is an optimal parameter selection problem involving a switched impulsive system with fixed jump times. Such problems can be solved using the software package MISER 3.3, which is based on gradient-descent optimization techniques.

In the next section, we demonstrate the efficiency of the proposed method outlined in this paper with a numerical example.

\section{Numerical Results}

We consider the shrimp farming problem formulated by Yu and Leung in [20]. The dynamics in this problem are described by the following differential equations:

$$
\begin{array}{lll}
\dot{x}_{1}(t)=-0.03 x_{1}(t), & x_{1}(0)=4.0 \times 10^{4}, \\
\dot{x}_{2}(t)=3.5-10^{-5} x_{1}(t) x_{2}(t), & x_{2}(0)=1,
\end{array}
$$

where

- $t$ is the time in weeks;

- $x_{1}(t)$ is the number of remaining shrimp at time $t$;

- $x_{2}(t)$ is the average weight of an individual shrimp in grams at time $t$. 


\begin{tabular}{|c||c|c|c|c|}
\hline Harvest No. & $x_{1}$ & $x_{2}$ & Harvest Time & Harvesting Fraction \\
\hline \hline 1 & 32173 & 6.86360 & 3.23733 & 0.113651 \\
\hline 2 & 27769 & 7.70245 & 3.94938 & 0.118238 \\
\hline 3 & 23778 & 8.67658 & 4.72935 & 0.123459 \\
\hline 4 & 20174 & 9.81421 & 5.58682 & 0.129454 \\
\hline 5 & 16933 & 11.15094 & 6.53325 & 0.136460 \\
\hline 6 & 14033 & 12.73232 & 7.58251 & 0.144774 \\
\hline 7 & 11452 & 14.61674 & 8.75118 & 0.154842 \\
\hline 8 & 9168 & 16.88034 & 10.05957 & 0.167346 \\
\hline 9 & 7163 & 19.62287 & 11.53240 & 0.183366 \\
\hline 10 & 6814 & 22.97670 & 13.20000 & 1.000000 \\
\hline
\end{tabular}

Table 1 Results from MISER 3.3 using the method described in [8]

Let $N$ denote the number of shrimp harvests and let $T$ denote the time of the final harvest. In this example, we take $T=13.2$ weeks.

Let $\tau_{i} \in[0, T]$ denote the time of the $i^{t h}$ harvest, with $\tau_{N}=T$ referring to the final harvest time. Note that these harvest times satisfy the following ordering constraints:

$$
0=\tau_{0} \leq \tau_{1} \leq \tau_{2} \leq \cdots \leq \tau_{N}=T .
$$

Furthermore, let $\zeta_{i}$ denote the fraction of shrimp stock harvested at time $\tau_{i}$. Then

$$
0 \leq \zeta_{i} \leq 1, \quad i=1, \ldots, N
$$

The first state variable, which represents the number of shrimp, is subject to the following jump conditions:

$$
x_{1}\left(\tau_{i}^{+}\right)-x_{1}\left(\tau_{i}^{-}\right)=-\zeta_{i} x_{1}\left(\tau_{i}^{-}\right), \quad \text { if } \quad i \in\{1, \ldots, N\} \text { and } \tau_{i}-\tau_{i-1} \geq \epsilon_{i},
$$

where $\epsilon_{i}>0$ is the given minimum duration between successive harvests. However, the second state variable, the average weight of shrimp, does not experience state jumps:

$$
x_{2}\left(\tau_{i}^{+}\right)-x_{2}\left(\tau_{i}^{-}\right)=0, \quad i=1, \ldots, N .
$$

The following model is suggested by Yu and Leung [20] for the total revenue over the production cycle of 13.2 weeks:

$$
R_{1}=\sum_{i=1}^{N}\left[\left(8 \times 10^{-3}\right) x_{1}\left(\tau_{i}^{-}\right) x_{2}\left(\tau_{i}\right) \zeta_{i}-50\right] .
$$

The above expression for total revenue assumes a sale price of $\$ 8$ per kilogram for the shrimp and a fixed cost of $\$ 50$ per harvest. Note that expression (37) for the revenue will result in the fixed cost of $\$ 50$ dollars being imposed at every harvest time, even if multiple harvest times coincide. Thus, we consider the modified revenue function given by

$$
R_{2}=\sum_{i=1}^{N} \widehat{\Phi}_{0, i}\left(\tau_{i}-\tau_{i-1}, x_{1}\left(\tau_{i}^{-}\right), x_{2}\left(\tau_{i}\right), \zeta_{i}\right),
$$

where $\widehat{\Phi}_{0, i}\left(\tau_{i}-\tau_{i-1}, x_{1}\left(\tau_{i}^{-}\right), x_{2}\left(\tau_{i}\right), \zeta_{i}\right)$ is defined as:

$$
\widehat{\Phi}_{0, i}\left(\tau_{i}-\tau_{i-1}, x_{1}\left(\tau_{i}^{-}\right), x_{2}\left(\tau_{i}\right), \zeta_{i}\right)= \begin{cases}\left(8 \times 10^{-3}\right) x_{1}\left(\tau_{i}^{-}\right) x_{2}\left(\tau_{i}\right) \zeta_{i}-50, & \text { if } \tau_{i}-\tau_{i-1} \geq \epsilon_{i}, \\ 0, & \text { if } \tau_{i}-\tau_{i-1}=0\end{cases}
$$

We assume here that $N=10$ (9 intermediate harvests and 1 final harvest).

The problem is to minimize $J=-R_{2}$, where $R_{2}$ is given by equation (38), subject to the dynamics (31)-(32), the jump conditions (35)-(36), the bounds (34) and the ordering constraints on $\tau_{i}$ given by (33). We first solve this problem using the method in [8], which is implemented in MISER 3.3 and involves maximizing the revenue function $R_{1}$. Here, we impose a lower bound of 0.45 weeks on each of the durations between two consecutive harvests. Note that this is required because of the nature of the problem class in [8], which does not allow zero subsystem durations.

The results obtained are tabulated in Table 1 . The optimal value of the revenue function corresponding to the results depicted in Table 1 is $2.9037867 \times 10^{3}$. Note that the solution in Table 1 


\begin{tabular}{|c||c|c|c|c|}
\hline Harvest No. & $x_{1}$ & $x_{2}$ & Harvest Time & Harvesting Fraction \\
\hline \hline 1 & 21535 & 7.77148 & 4.27134 & 0.388030 \\
\hline 2 & 10580 & 12.64344 & 7.80877 & 0.453674 \\
\hline 3 & 9000 & 22.23958 & 13.20000 & 1.000000 \\
\hline
\end{tabular}

Table 2 Results from our new method described in this paper

\begin{tabular}{|c||c|c|c|}
\hline$\sigma$ & $\lambda^{*}$ & Penalty Function Value & Constraint Violation \\
\hline \hline $10^{\sigma}$ & $1.00000 \times 10^{-1}$ & $3.19832213 \times 10^{3}$ & $8.80240 \times 10^{-2}$ \\
\hline $10^{1}$ & $1.00000 \times 10^{-1}$ & $3.19806847 \times 10^{3}$ & $9.10594 \times 10^{-2}$ \\
\hline $10^{2}$ & $1.00000 \times 10^{-1}$ & $3.19553193 \times 10^{3}$ & $3.03930 \times 10^{-3}$ \\
\hline $10^{3}$ & $1.18314 \times 10^{-3}$ & $3.18890576 \times 10^{3}$ & $1.89485 \times 10^{-9}$ \\
\hline $10^{4}$ & $3.53827 \times 10^{-6}$ & $3.18893349 \times 10^{3}$ & $1.87380 \times 10^{-16}$ \\
\hline $10^{5}$ & $1.75704 \times 10^{-6}$ & $3.18893339 \times 10^{3}$ & $4.62264 \times 10^{-17}$ \\
\hline $10^{6}$ & $3.92612 \times 10^{-7}$ & $3.18893340 \times 10^{3}$ & 0.00000 \\
\hline
\end{tabular}

Table 3 Numerical convergence using $\alpha=2.00$ and $\beta=1.55$

includes every potential harvest (i.e. no harvests have been deleted). This is expected, as the algorithm in [8] has no capacity to eliminate non-optimal harvests. Thus, the solution in Table 1 may or may not be optimal.

Table 2 shows the results obtained using our new algorithm developed in this paper. The notable feature here is that, by imposing a minimum duration of 0.45 weeks between two consecutive harvests, we obtained a maximum revenue of $3.1889334 \times 10^{3}$ with only 3 harvests taking place. In essence, in this example, 7 harvests have been removed (recall that we allowed up to $N=10$ potential harvests).

The optimal harvest times corresponding to the solution in Table 2 are as follows:

$$
\begin{gathered}
\tau_{1}=4.27134, \quad \tau_{2}=4.27134, \quad \tau_{3}=4.27134, \quad \tau_{4}=4.27134, \quad \tau_{5}=7.80877, \\
\tau_{6}=7.80877, \quad \tau_{7}=7.80877, \quad \tau_{8}=7.80877, \quad \tau_{9}=7.80877, \quad \tau_{10}=13.2 .
\end{gathered}
$$

Table 3 shows the progression of the penalty method corresponding to the solution in Table 2. Note that these results show a clear convergence of the objective function and the constraint violation as the penalty parameter $\sigma$ is increased.

Figure 1 shows the number of shrimps at each point in the production cycle for the solutions in Tables 1 and 2. Note the difference in the solutions in Figure 1, one of which was obtained using the method in [8] and the other using our new method. As there are no jump conditions imposed on the average weight of the shrimp (see (36)), the two trajectories depicted in Figure 2 are almost the same.

\section{Concluding Remarks}

We have developed an effective computational technique for solving a class of impulsive switched system optimal control problems, where the objective and constraints depend on the duration of each subsystem. This technique is based on a novel combination of the time-scaling transformation, binary relaxation, and exact penalty methods. The algorithm was successfully tested on a shrimp farming problem arising in aquaculture operations.

\section{References}

1. C. Y. F. Ho, B. W. K. Ling, Y. Q. Liu, P. K. S. Tam, and K. L. Teo, Optimal PWM control of switched-capacitor DC-DC power converters via model transformation and enhancing control techniques. IEEE Transactions on Circuits and Systems I: Regular Papers, 55(5), 1382-1391 (2008)

2. L. S. Jennings, M. E. Fisher, K. L. Teo, and C. J. Goh, MISER 3.3 Optimal Control Software: Theory and User Manual. University of Western Australia, Perth (2004)

3. C. Jiang, Q. Lin, C. Yu, K. L. Teo, and G. R. Duan, An exact penalty method for free terminal time optimal control problem with continuous inequality constraints. Journal of Optimization Theory and Applications, 154(1), 30-53 (2012)

4. C. Jiang, K. L. Teo, R. Loxton, and G. R. Duan, A neighboring extremal solution for an optimal switched impulsive control problem. Journal of Industrial and Management Optimization, 8(3), 591-609 (2012) 


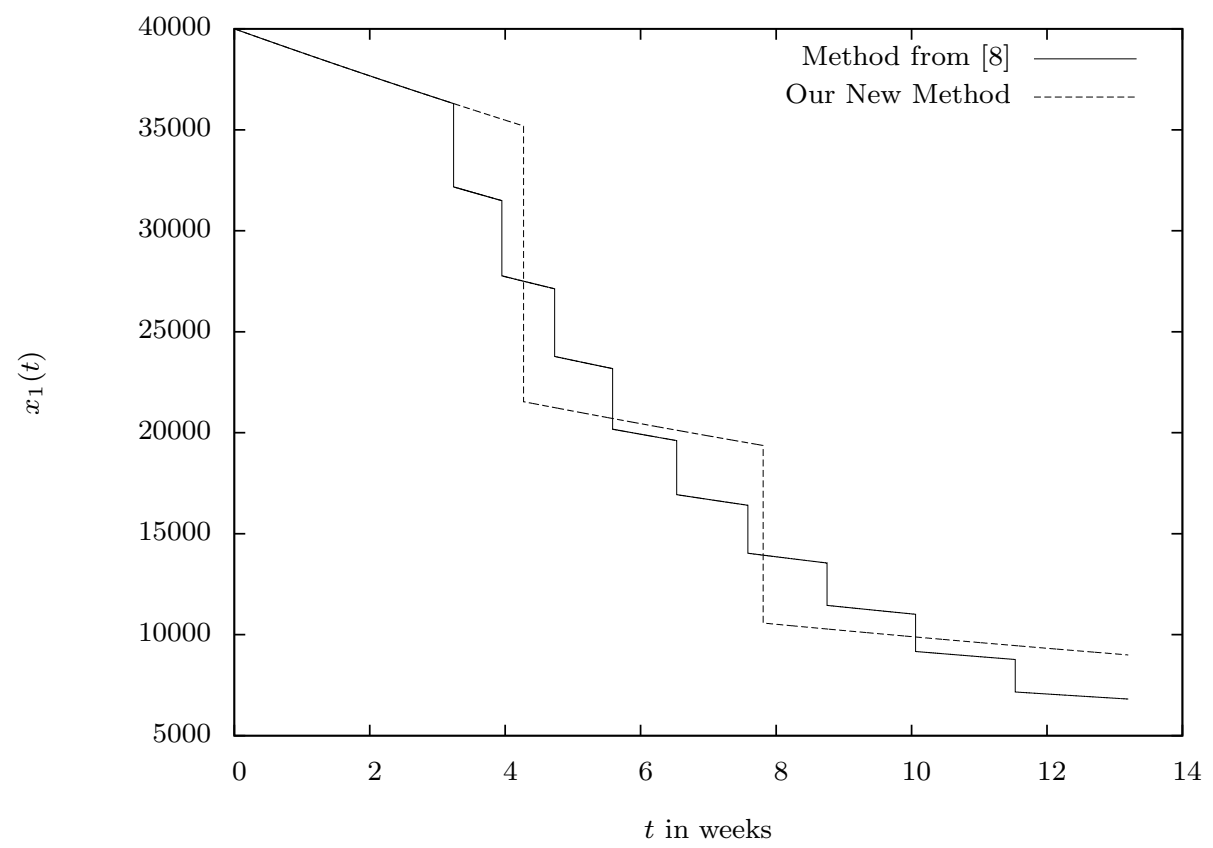

Fig. 1 Number of Shrimps

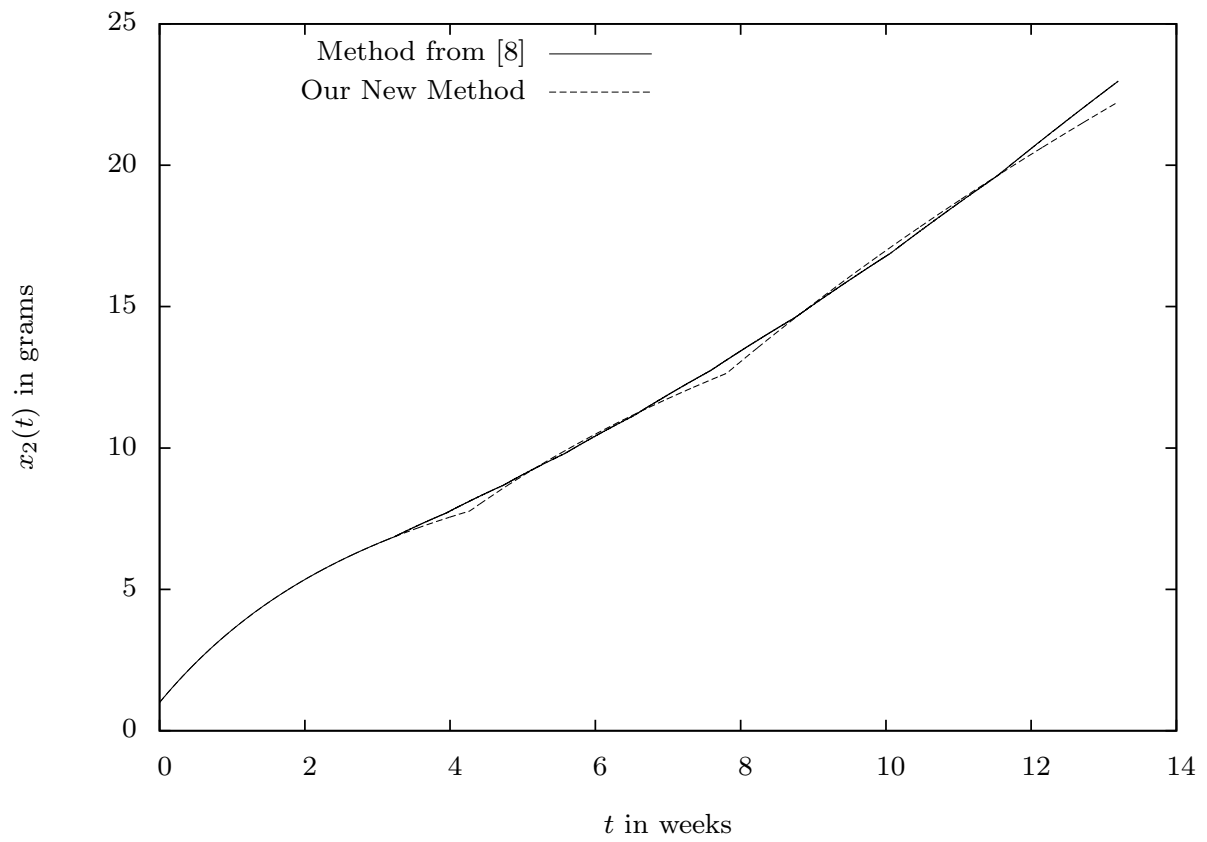

Fig. 2 Average Weight of Shrimp

5. A. Khadra, X. Liu, and X. Shen, Application of impulsive synchronization to communication security. IEEE Transactions on Circuits and Systems I: Fundamental Theory and Applications, 50(3), 341-351 (2003)

6. Q. Lin, R. Loxton, and K. L. Teo, The control parameterization method for nonlinear optimal control: A Survey. Journal of Industrial and Management Optimization, accepted

7. Q. Lin, R. Loxton, K. L. Teo, and Y. H. Wu, A new computational method for a class of free terminal time optimal control problems. Pacific Journal of Optimization, 7(1), 63-81 (2011)

8. Q. Lin, R. Loxton, K. L. Teo, and Y. H. Wu, A new computational method for optimizing nonlinear impulsive systems. Dynamics of Continuous, Discrete and Impulsive Systems: Series B - Applications and Algorithms, 18(1), 59-76 (2011) 
9. Q. Lin, R. Loxton, K. L. Teo, Y. H. Wu, and C. Yu, A new exact penalty method for semi-infinite programming problems. Journal of Computational and Applied Mathematics, submitted

10. Y. Liu, K. L. Teo, L. S. Jennings, and S. Wang, On a class of optimal control problems with state jumps. Journal of Optimization Theory and Applications, 98(1), 65-82 (1998)

11. R. Loxton, K. L. Teo, and V. Rehbock, Computational method for a class of switched system optimal control problems. IEEE Transactions on Automatic Control, 54(10), 2455-2460 (2009)

12. R. C. Loxton, K. L. Teo, V. Rehbock, and W. K. Ling, Optimal switching instants for a switched-capacitor DC/DC power converter. Automatica, 45(4), 973-980 (2009)

13. D. G. Luenberger and Y. Ye, Linear and Nonlinear Programming, 3rd edition. New York, Springer (2008)

14. J. Nocedal and S. J. Wright, Numerical Optimization, 2nd edition. New York, Springer (2006)

15. Y. Peng and X. Xiang, Second order nonlinear impulsive time-variant systems with unbounded perturbation and optimal controls. Journal of Industrial and Management Optimization, 4(1), 17-32 (2008)

16. T. Ruby, V. Rehbock, and W. B. Lawrance, Optimal control of hybrid power systems. Dynamics of Continuous, Discrete and Impulsive Systems: Series B - Applications and Algorithms, 10(3), 429-439 (2003)

17. W. Wei, K. L. Teo, and Z. Zhan, A numerical method for an optimal control problem with minimum sensitivity on coefficient variation. Applied Mathematics and Computation, 218(4), 1180-1190 (2011)

18. C. Z. Wu and K. L. Teo, Global impulsive optimal control computation. Journal of Industrial and Management Optimization, 2(4), 435-450 (2006)

19. H. Xu and K. L. Teo, $H_{\infty}$ optimal stabilization of a class of uncertain impulsive systems: An LMI approach. Journal of Industrial and Management Optimization, 5(1), 153-159 (2009)

20. R. Yu and P. Leung, Optimal partial harvesting schedule for aquaculture operations. Marine Resource Economics, 21(3), 301-315 (2006)

21. C. Yu, K. L. Teo, and Y. Bai, An exact penalty function method for nonlinear mixed discrete programming problems. Optimization Letters, 7 (1), 23-38 (2013)

22. C. Yu, K. L. Teo, L. Zhang, and Y. Bai, A new exact penalty function method for continuous inequality constrained optimization problems. Journal of Industrial and Management Optimization, 6(4), 895-910 (2010)

23. C. Yu, K. L. Teo, L. Zhang, and Y. Bai, On a refinement of the convergence analysis for the new exact penalty function method for continuous inequality constrained optimization problem. Journal of Industrial and Management Optimization, 8(2), 485-491 (2012) 